\title{
Radio-continuum spectrum, brightness temperature, and planetary nebulae properties
}

\author{
R. Gruenwald and A. Aleman
}

\author{
IAG-USP, Rua do Matão, 1226, 05508-090 São Paulo, Brazil \\ e-mail: ruth@astro.iag.usp.br
}

Received 30 May 2006 / Accepted 18 September 2006

ABSTRACT

\begin{abstract}
Context. Radio-continuum spectra are frequently used to infer the density distribution of ionized gaseous regions, while observed correlations between the brightness temperature and other distance-independent parameters are used to test evolutionary models of planetary nebulae.

Aims. We check empirical correlations among features derived from the observed radio data and the inferred conclusions available in the literature, using self-consistent photoionization models.

Methods. Photoionization models are computed for the physical conditions of planetary nebulae in order to derive self-consistent radio-continuum spectra, as well as the brightness temperature.

Results. The temperature and ionization distributions throughout the nebulae explain the observed range of spectral indexes in the thick region of the spectrum, even for a uniform density distribution, usually challenged in the literature. The obtained models fit the observed radio spectra for planetary nebulae in a large range of spectral indexes. Our calculations show a correlation between the spectral index obtained in a given frequency range and the nebula size, as well as reproduce the observed relations between the brightness temperature and other distance-independent parameters. Such diagrams are frequently used to check evolutionary models of the central star and/or of the nebula.

Conclusions. Since PNe images clearly show that the density is not constant inside the nebulae, and models with uniform and nonuniform density distributions can both reproduce the observed radio spectra, we conclude that it is not possible to favour one of them from the radio data, or to infer a particular density distribution for planetary nebulae.
\end{abstract}

Key words. ISM: planetary nebulae: general - radio-continuum: ISM

\section{Introduction}

The spectral distribution of the observed radio continuum emission in many compact ionized regions shows a weak dependence on the frequency and can present a turn-over at low frequencies. The observed radiation is believed to be primarily due to thermal free-free emission from the ionized gas. Following the theory, the radiation at high frequencies from an isothermal, constant density ionized region corresponds to low optical depth emission where the flux has a slight dependence on the frequency $\left(F_{v} \propto v^{-0.1}\right)$. In the limit of high opacities the spectrum increases with the frequency with a power-law index equal to +2 . Radio observations of planetary nebulae (PNe) and other ionized regions show that for frequencies greater than $5 \mathrm{GHz}$ the spectra generally correspond to the optically thin case.

For many objects presenting turn-over frequencies in the observed range, the spectral index calculated at the thick part of the low-frequency spectrum is, however, much lower than the theoretical value of +2 . Such spectra have been generally explained in the literature by isothermal regions with particular density distributions, as presented in the following.

For an isothermal envelope with a power-law electron density distribution there is a relationship between the shape of the density distribution and the power-law index of the radio continuum spectrum (see, for example, Wright \& Barlow 1975). For a constant velocity mass flow the density distribution is $\alpha r^{-\beta}$, with $\beta=2$, leading to a radio power-law index $\alpha=0.6$, assuming that the gas is $100 \%$ ionized. For the last 30 years, the discussion about the power-law index of the radio continuum and some characteristic parameters of ionized regions has been mainly focused on explaining the values between 0.6 (unbounded wind shell model) and 2 (optically thick free-free emission) deduced from the observations.

The discussion started with Seaquist \& Gregory (1973) who analyzed the radio continuum emission of the symbiotic star V1016 Cygni, obtaining $\alpha=0.75$ and $\beta=2.14$, which approaches the value of 2.0 expected for a uniform mass outflow at constant velocity. A more detailed treatment was done by Wright \& Barlow (1975) for several early-type stars, assuming an unbounded isothermal envelope produced by a uniform and steady mass loss. In order to explain the observed range of spectral indexes (0.6 to 1.15), Wright \& Barlow discussed some effects that could cause the deviation from 0.6 , for example, variability due to non-uniform mass loss rates and the presence of neutral condensations. The radio continuum spectrum from spherical extended envelopes with electron density $\propto r^{-\beta}$ was also analyzed by Panagia \& Felli (1975), while Olnon (1975) analyzed the radio spectra produced by regions with various density distributions. The radio spectra of Vy2-2 and $\mathrm{Hb} 12$ (later classified as planetary nebulae) as well as of V1016 Cyg have been studied by Marsh (1975) who, in order to explain spectral indexes greater than 0.6, introduced a finite outer radius for the ionized region produced by a steady flow with uniform velocity. Using numerical simulations Viner et al. (1979) analyzed the radio 
continuum and recombination line emission for spherically symmetric HII regions with radial variation in temperature, electron density, velocity and turbulence. Applying the results to the planetary nebula NGC 7027, the authors favour a density gradient model, although Pottasch (1984) suggests a constant density for the same planetary. Contradictory results were also found for Vy2-2. Marsh (1975) and Seaquist \& Davis (1983) proposed a wind shell model with $\beta=2$, while Pottasch (1984) obtained a power-law index with $\beta=3.5$, and Harris \& Scott (1976) concluded that a uniform density slab could explain the power-law spectral index equal to 2 .

Several compact planetary nebulae were studied by Taylor et al. (1987). Defining the spectral index at the point in the spectrum where the flux equals one-half of the peak optically thin flux density, they explained the range of observed spectral indexes ( $\alpha=0.6$ to 1.8 ) by radiation- bounded isothermal nebulae with density distribution $\propto r^{-\beta}$. Their fit suggests an average in$\operatorname{dex} \beta=2$, consistent with a stellar wind at constant mass-loss rate and velocity. They also found an anti-correlation between their measured spectral index and the nebula size, concluding that this result suggests an evolutionary trend in which older nebulae are more inhomogeneous. Such a conclusion was based on the assumptions that older nebulae are larger and that high values for alpha $(\sim 2)$ correspond to uniform density nebulae, while density inhomogeneities produce lower spectral indexes.

As well known from photoionization models, a uniform density distribution does not necessarily imply constant ionic and electronic distributions, which depend on the radiation transfer inside the nebula. Photoionization codes have been used to reproduce the observed radio emission of planetary nebulae by Middlemass (1990) and Dudziak et al. (2000). The radio spectrum of NGC 7027 was reproduced by Middlemass (1990), who adopted a density distribution obtained from the radio profile. Two PNe in the Sagittarius dwarf galaxy were studied by Dudziak et al. (2000) who reproduced reasonably well the fluxes for two observed frequencies in the thin spectral region for one object; problems with the fitting for the spectrum of a second object, with fluxes measured in three radio frequencies, were justified by arguing that radio flux predictions are sensitive to details of the geometry.

More recently, with simplified isothermal models Siodmiak $\&$ Tylenda (2001) analyzed a large number of PNe in two frequencies and concluded that an $r^{-2}$ density distribution is not typical of PNe. These authors also concluded that a reasonable representation of the observations can be obtained with a twocomponent model where the two regions have different optical thickness.

Another important issue is the possibility of testing evolutionary models for planetary nebulae using parameters estimated from radio observations. The behaviour of the radio continuum brightness temperature $\left(T_{\mathrm{b}}\right)$ with other parameters, like gas density or the size of the ionized nebula, has been used to test evolutionary models and to distinguish the more appropriate evolutionary track (Kwok 1985; Zhang \& Kwok 1993; Van de Steene \& Zijlstra 1995; Zhang 1995).

We obtain, in the present paper, radio continuum spectra of photoionized nebulae using self-consistent temperature as well as electronic and ionic density distributions inside the gas, provided by detailed photoionization models. The set of models represents a typical range of stellar and nebular parameters for PNe. Planetary nebulae were chosen since the ionizing source and corresponding spectrum are reasonable well-known, providing a reliable determination of the physical conditions along the nebulae. The resulting radio spectrum and the corresponding brightness temperature are obtained for each model. These spectra are compared with those obtained with constant temperature and ionic abundances. Some of the correlations commonly used in the literature for obtaining information about physical characteristics and evolutionary models of PNe are analyzed and discussed. In Sect. 2 the models and the adopted input parameters are described, while in Sect. 3 some general results are presented and discussed. An application of the models to six planetary nebulae with well observed spectra is also presented (Sect. 4). Conclusions are outlined in Sect. 5.

\section{Models}

The models were obtained with the photoionization code Aangaba (Gruenwald \& Viegas 1992) applied for typical conditions in PNe. The physical conditions of the gas are determined by solving the coupled equations of ionization and thermal balance for a spherically symmetric cloud. Several processes of ionization and recombination, as well as of gas heating and cooling, are taken into account. The energy input into the gas is essentially due to ionization of atoms and atomic ions by primary (stellar) and secondary (diffuse) radiation. The processes contributing to the gas cooling are collisional excitation, radiative and dielectronic recombination, thermal ionization, and free-free emission. The transfer of the primary and diffuse radiation fields is treated in the outward-only approximation. The effect of dust on the ionizing radiation is not taken into account. Twelve elements $(\mathrm{H}, \mathrm{He}, \mathrm{C}, \mathrm{N}, \mathrm{O}, \mathrm{Mg}, \mathrm{Ne}, \mathrm{Si}, \mathrm{S}, \mathrm{Ar}, \mathrm{Cl}$, and $\mathrm{Fe})$ are included.

The calculation of the radio-continuum emissivity is based on the equations for the free-free mechanism (see, for example, Rybicki \& Lightman 1979), with the appropriate expression for the Gaunt factor in the radio spectral region, which has a slight dependence on the frequency (see, for example, Osterbrock 1989); for high photon to gas energy ratios, the formula given by Seaton (1960) is used. The radio emissivity at each point of the nebula is obtained, as well as the intensity emitted by the whole nebula, taking into account the absorption of the radiation as it crosses the nebula.

The emitted radio spectrum and the brightness temperature were obtained from models assuming stellar and nebular parameters typical of PNe. The central star emits as a black-body with temperature and luminosity in the range $2 \times 10^{4}$ to $2 \times 10^{5} \mathrm{~K}$ and 30 to $2 \times 10^{4} L_{\odot}$. A typical chemical abundance for PNe (Kingsbourgh \& Barlow 1994) is assumed for the gas, which includes $\mathrm{He}, \mathrm{C}, \mathrm{N}, \mathrm{O}, \mathrm{Ne}, \mathrm{S}$, and Ar. For the remaining elements $(\mathrm{Mg}, \mathrm{Si}, \mathrm{Cl}$, and $\mathrm{Fe}$ ) a value equal to a hundredth solar is adopted in order to take into account their presence in grains (Stasinska \& Tylenda 1986). The gas density is in the range $10^{2}$ to $10^{6} \mathrm{~cm}^{-3}$. For completely optically thick models at the Lyman limit the calculation stops at $R_{\max }$, where the fraction of neutral hydrogen is $0.01 \%$. The emitted spectra were obtained after an integration of the intensity over the projected area for nebulae with different optical depths at the hydrogen threshold. In order to compare the brightness temperature with the observations, this temperature was calculated from the total luminosity in $5 \mathrm{GHz}$ emitted by the nebula and $R_{\max }$.

For comparison, simplified models for isothermal nebulae with constant electron and ionic densities are also calculated. For each photoionization model (with a given $T_{*}, L_{*}$, and gas density), a corresponding simplified model is obtained. For this, it is assumed that the gas consists of $\mathrm{H}$ and $\mathrm{He}$, and the nebula size, the gas temperature, as well as the $\mathrm{H}$ and He ionic fraction are given, respectively, by $R_{\max }$, the average temperature of the 
$\mathrm{H}^{+}$region, and mean values, all given by the corresponding photoionization model. In both kinds of models the internal radius was assumed equal to $10^{15} \mathrm{~cm}$; the exact value for this parameter does not affect our conclusions since for an optically thick model increasing the internal radius leads to increasing $R_{\max }$, with the total emitting volume slightly decreasing.

\section{Results}

From the literature described in Sect. 1, it is clear that the main physical characteristic challenged by the radio spectral indexes obtained from the observations is a uniform density distribution. Therefore, this assumption is the first to be discussed below. The claims are based on the part of the radio spectra that corresponds to the optically thick case, for which the spectral index should be +2 . Except simplifying the calculations, a uniform density distribution does not necessarily correspond to constant ionic and electronic distributions. In addition, as it will be discussed below, the estimation of the radio power-law index from the observations may differ from object to object, and even for the same object, since the frequency range used may differ from an author to another.

\subsection{The radio spectrum and the gas density}

The emitted radio spectra obtained from photoionization models adopting a star with $T_{*}=10^{5} \mathrm{~K}$ and $L_{*} / L_{\odot}=3000$, for nebulae with different values of the gas density, are shown in Fig. 1a. The theoretical flux (solid lines) is normalized for a distance of $1 \mathrm{kpc}$ after integration of the specific intensity over the nebula area. In the plot, all models correspond to optically thick nebulae at the $\mathrm{H}$ threshold, and are labelled by the logarithm of the gas density. The dotted, dashed and dot-dashed lines connect points for which the optical depth of the nebula along the line of sight passing by the central star is, respectively, $0.5,1.0$, or 5.0 for the corresponding frequencies. From the figure, it can be seen that, as expected, the higher the density the higher the turn-over frequency, as well as the frequency corresponding to a given value of the gas optical depth. For example, for a hydrogen density $n_{\mathrm{H}}=10^{3} \mathrm{~cm}^{-3}$ the optical depth is greater than 1 for frequencies lower than $0.3 \mathrm{GHz}$, while for $n_{\mathrm{H}}=10^{4} \mathrm{~cm}^{-3}$ the corresponding frequency is $1.2 \mathrm{GHz}$. For each model the frequency for which the flux is half the maximum has an optical depth of the order of 5 .

The gas density is the main parameter defining the turn-over frequency. The measured flux from a given nebula depends, evidently, on the number of ionizing photons $\left(Q_{\mathrm{H}}\right)$ and on its distance. For the adopted range of stellar temperatures, the effect of $T_{*}$ on $Q_{\mathrm{H}}$ is small.

The emitted radio spectra of photoionization models are compared with those obtained for constant temperature and ionic density nebulae in Fig. 1b. As described above, the simplified model to be compared to a given photoionization model is obtained assuming a gas temperature and ionic density constant and equal to mean values, as given by the corresponding photoionization model. The nebula size is the same for both models. The spectra calculated by the photoionization and by the simplified models are compared for three different densities. The input parameters used are the same as in Fig. 1a. The small shifts in the upper part of the spectra obtained from the simplified models are due to differences in the gas temperature. Notice that spectra for models with constant fractional ionization and constant gas temperature are steeper than those for the corresponding
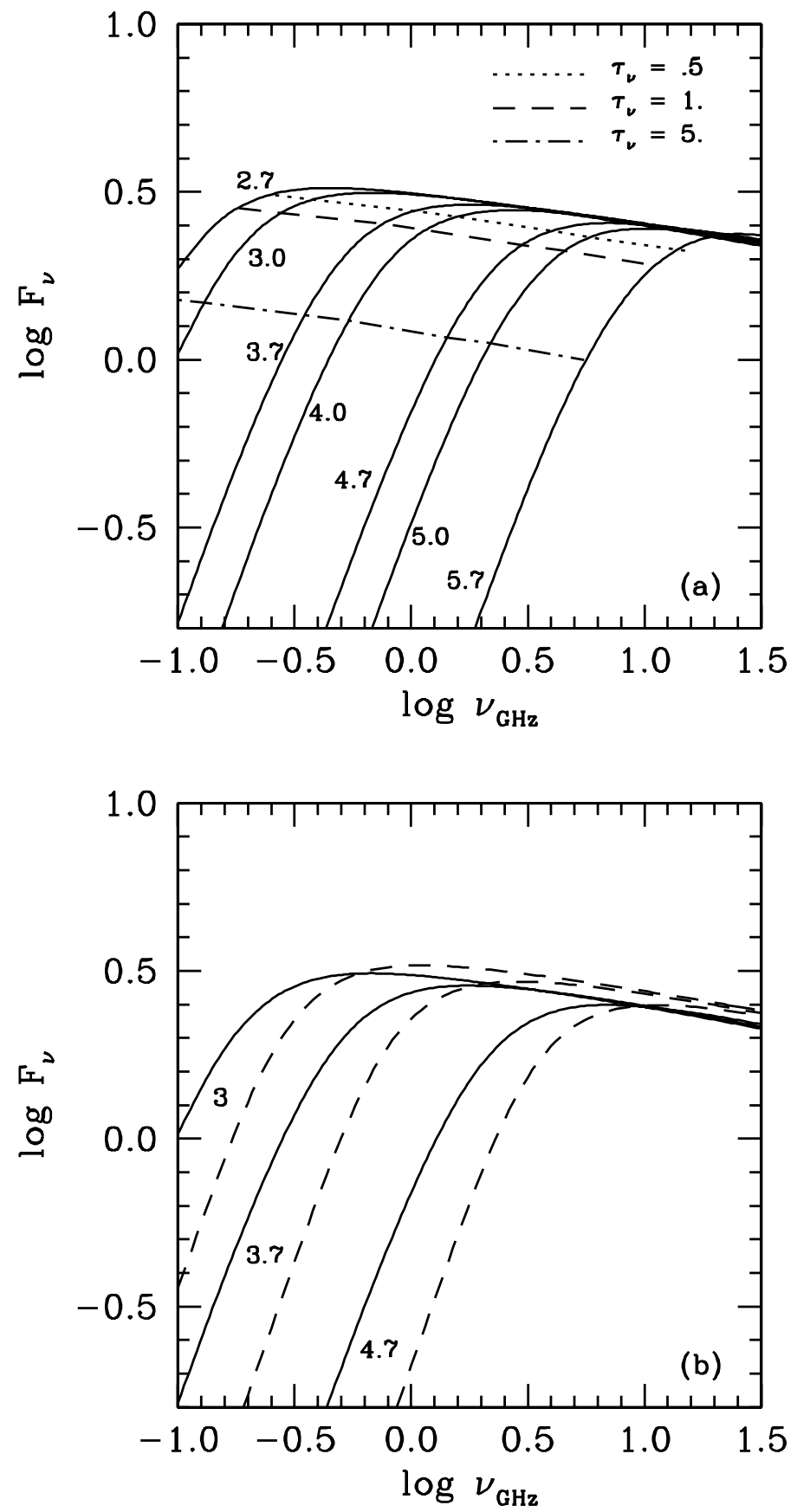

Fig. 1. Radio continuum spectra from photoionization models (solid lines) for $T_{*}=10^{5} \mathrm{~K}$ and $L_{*} / L_{\odot}=3000$, normalized to a distance of $1 \mathrm{kpc}$. The flux is given in Jy and the frequency in GHz; the curves are labelled with the logarithm of the gas density. a) Dotted, dashed and dot-dashed lines connect points for optical depths equal to $0.5,1.0$, and 5.0, in the corresponding frequencies; b) the dashed lines correspond to simplified models with constant temperature and ionic density, and are labelled with the logarithm of the gas density.

photoionization model in the optically thick part of the radio spectrum.

For a given ionizing spectrum and a given frequency range, the spectral power-law index in the optically thick part of the radio spectra is steeper for higher densities (Figs. 1a and b). Furthermore, for a given gas density, the spectrum is steeper for simplified models (Fig. 1b). Such behaviour can be explained by the ionization structure in the nebula. In fact, for low density regions the variation of the ionic abundance is not as sharp 


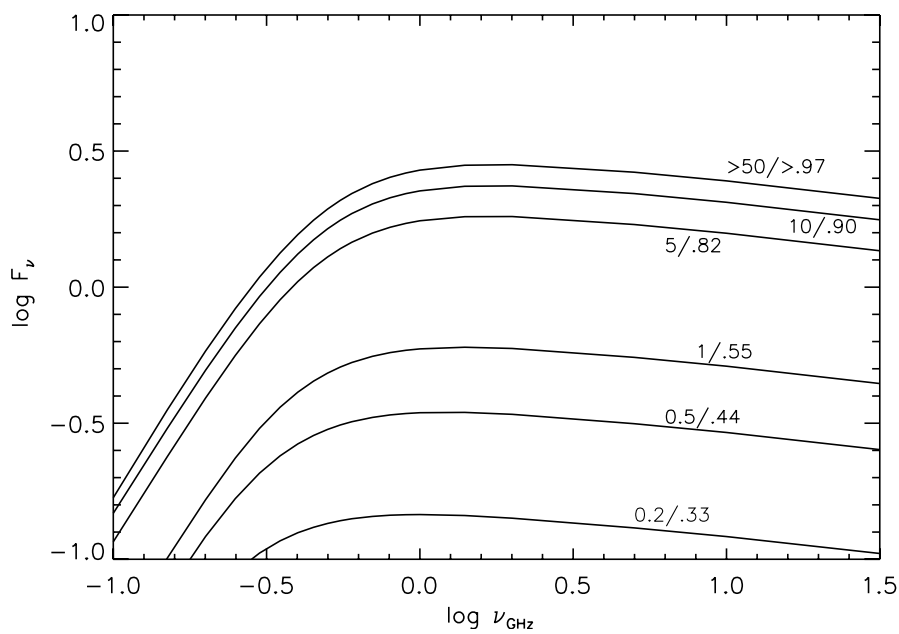

Fig. 2. Radio spectra for nebulae with $T_{*}=10^{5} \mathrm{~K}, L_{*} / L_{\odot}=3000$, $n_{\mathrm{H}}=5000 \mathrm{~cm}^{-3}$, and different optical depths at the Lyman limit. Numbers at the side of each curve are the corresponding optical depth at the $\mathrm{H}$ threshold and the fraction of $R_{\max }$; units are the same as in Fig. 1.

as for high density nebulae. For these, the variation of the ionic abundance mimics the one for a constant density nebula.

For each optically thick model, we also calculated the corresponding radio spectra for several optical depths at the Lyman limit. These spectra are illustrated in Fig. 2 by the results of models with $T_{*}=10^{5} \mathrm{~K}, L_{*} / L_{\odot}=3000$ and $n_{\mathrm{H}}=5000 \mathrm{~cm}^{-3}$. The curves are labelled with the optical depth at the Lyman limit and the corresponding fraction of $R_{\max }$.

The degree of clumpiness in several planetary nebulae was estimated by Terzian \& Dickey (1973) from radio measurements, and the authors remark that for inhomogeneous regions the resulting spectrum should present more than a turn-over frequency. We obtained simple composite models, with more than one value for the density, and the results show that, depending on the density ratio and on the degree of clumpiness, the change from the optically thin regime to the thick one can be much smoother than that for the constant density model, or even present more than a turn-over frequency.

\subsection{The radio power-law index}

In order to discuss the behaviour of the spectral index of the optically thick part of the radio spectrum with the gas density and with the size of the ionized region, we adopted a frequency interval 0.4 to $0.6 \mathrm{GHz}$. This choice was due to the fact that this frequency range lies in the thick part of the spectra of many PNe, and many observations and surveys have been made at $408 \mathrm{MHz}$ and at frequencies around $600 \mathrm{MHz}$.

The results are shown in Fig. 3. Solid lines correspond to models with $T_{*}=10^{5} \mathrm{~K}$ and three values for $L_{*}$. The dependence of the radio index with the gas density is shown in Fig. 3a, while that with the nebula size $(R)$ appears in Fig. $3 b$. The results correspond to radiation-bounded nebulae, where the nebula size is given by the radius where hydrogen is .01\% ionized $\left(R_{\max }\right)$. For each value of the stellar luminosity, the size was calculated by the models, varying the density. Results for models with a given $L_{*}$ and different values for $T_{*}$ differ by up to $15 \%$. Notice that spectra corresponding to a given stellar temperature and a given gas density (consequently, a given $R$ ) present different power-law indexes for the chosen frequency range depending on the stellar luminosity, being steeper for higher $L_{*}$. This
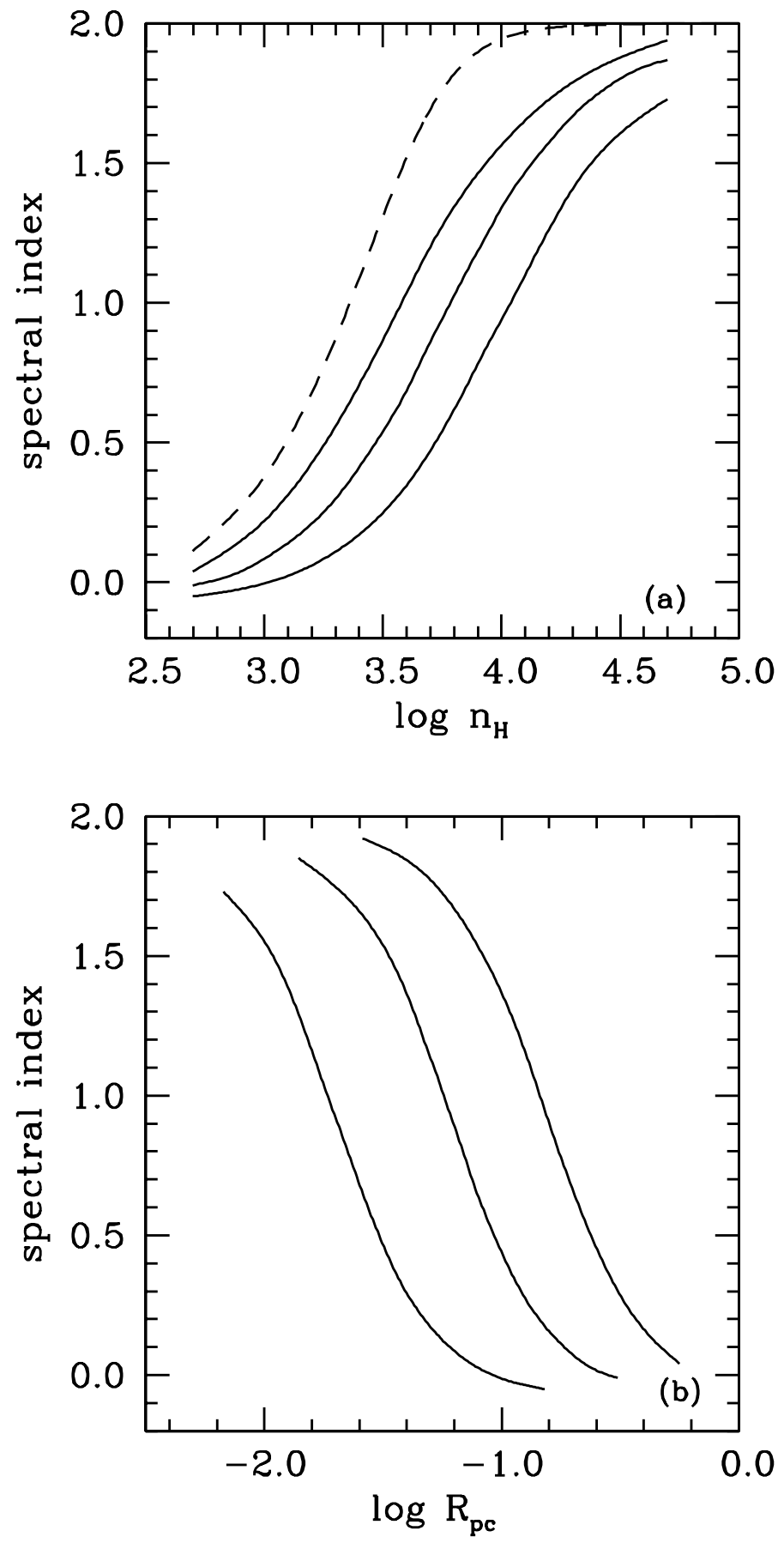

Fig. 3. Spectral index for models with $T_{*}=10^{5} \mathrm{~K}$ : a) as a function of the gas density; solid lines show the spectra from optically thick photoionization models with $L_{*} / L_{\odot}=20000$ (upper curve), 3000 (middle), and 300 (lower), while the dashed line corresponds to a simplified model with $L_{*} / L_{\odot}=3000$; b) as a function of the nebula size, for photoionization models with $L_{*} / L_{\odot}=20000$ (upper curve), 3000 (middle), and 300 (lower). The spectral index corresponds to the $0.4-0.6 \mathrm{GHz}$ frequency range.

is due to the change on the ionic distribution inside the nebula which leads to a change in the optical depth. The dashed line in Fig. 3a illustrates the results of a simplified model corresponding to a photoionization model with $L_{*} / L_{\odot}=3000$. From this figure, it is clear that detailed models can present indexes much lower than 2. Regarding the simplified models, a steeper increase of the power index with the density is found, and values closer 
to +2 can be obtained for a larger range of densities. Such correlations can be easily understood from photoionization models, in terms of the gas density. As shown above, the density is the main parameter that defines the behaviour of the resulting radio spectrum. Since for a given ionizing star (more exactly, for a given number of ionizing photons) the nebula density also defines the size of the ionized region, a correlation between the radio index and the density is expected.

An inverse correlation between the turn-over frequency and the spectral index of the thick part of the radio spectrum was obtained by Aaquist \& Kwok (1991), for seven compact nebulae. This is the opposite of what it would be expected from the results presented above, since higher densities imply higher turnover frequencies and also higher spectral indexes. Notice, however, that the turn-over frequencies were determined by those authors by the cross-over of the line representing the thin part of the spectrum (with a power-law index fixed at -0.1) and the line representing the thick part of the spectrum, which was fitted with one spectral index. As shown in Fig. 1a, for a given nebula a different spectral index can be obtained, depending on the chosen spectral region.

\subsection{The radio brightness}

The radio continuum brightness temperature, $T_{\mathrm{b}}$, is a distanceindependent parameter, relatively easy to measure. Since most $\mathrm{PNe}$ are optically thin at $5 \mathrm{GHz}$, the brightness temperature at this frequency has been used to derive some PNe properties. For example, an empirical relationship between $T_{\mathrm{b}}$ and the gas density has been used to test models of the dynamic evolution of PNe (Kwok 1985; Zhang \& Kwok 1993), while a plot of $T_{\mathrm{b}}$ versus $T_{*}$ has been used to check evolutionary model tracks and to derive the core mass as well as the stellar luminosity for individual nebulae (Zhang \& Kwok 1993). From a large sample of PNe with known distances, Van de Steene \& Zijlstra (1995) and Zhang (1995) obtained a correlation between $T_{\mathrm{b}}$ and the nebula size, proposing a new statistical distance scale for PNe and checking some evolutionary tracks.

The radio-continuum brightness temperature in $5 \mathrm{GHz}\left(T_{\mathrm{b}}\right)$ as a function of the gas density, the nebula radius, and $T_{*}$ is shown in Figs. $4 \mathrm{a}-\mathrm{c}$, respectively. In these figures, the curves correspond to optically thick photoionization models at the Lyman limit, while the points are obtained from the literature for observed PNe (Zhang \& Kwok 1993; Zhang 1995). For these, the gas density is given in terms of the electron density. The different curves presented in Figs. $4 \mathrm{a}$ and $\mathrm{b}$ correspond to models with different values of $L_{*}$ and the same value of $T_{*}=10^{5} \mathrm{~K}$; higher curves correspond to higher $L_{*}$. The solid lines in Fig. 4c show the results from models with $n_{\mathrm{H}}=5000 \mathrm{~cm}^{-3}$ and different values for $L_{*}$ (higher curves corresponding to higher $L_{*}$ ); dashed lines give the results for $n_{\mathrm{H}}=100$ and $50000 \mathrm{~cm}^{-3}$ (the higher the curve the higher the gas density), adopting $L_{*} / L_{\odot}=3000$. In Figs. $4 \mathrm{a}$ and $\mathrm{b}$ it can be seen that for very high densities the curves tend to a single value of $T_{\mathrm{b}}$, corresponding to densities for which the nebulae are thick at $5 \mathrm{GHz}$.

The main effect is due to the gas density (Fig. 4a); such an effect is reflected in the behaviour of $T_{\mathrm{b}}$ with the nebula size (Fig. 4b). For example, for $T_{*}=10^{5} \mathrm{~K}$ and $L_{*} / L_{\odot}=3000$, the brightness temperature varies by four orders of magnitude in the range $n_{\mathrm{H}}=10^{2}$ to $10^{5} \mathrm{~cm}^{-3}: T_{\mathrm{b}}=1.3$ to $11700 \mathrm{~K}$. Regarding the effect of the stellar luminosity, for $T_{*}=10^{5} \mathrm{~K}$ and $n_{\mathrm{H}}=5000 \mathrm{~cm}^{-3}$, the brightness temperature is $T_{\mathrm{b}}=44$, 113,263 , and $512 \mathrm{~K}$, for $L_{*} / L_{\odot}=30,300,3000$, and 20000 . On the other hand, the dependence of $T_{\mathrm{b}}$ on the stellar temperature is
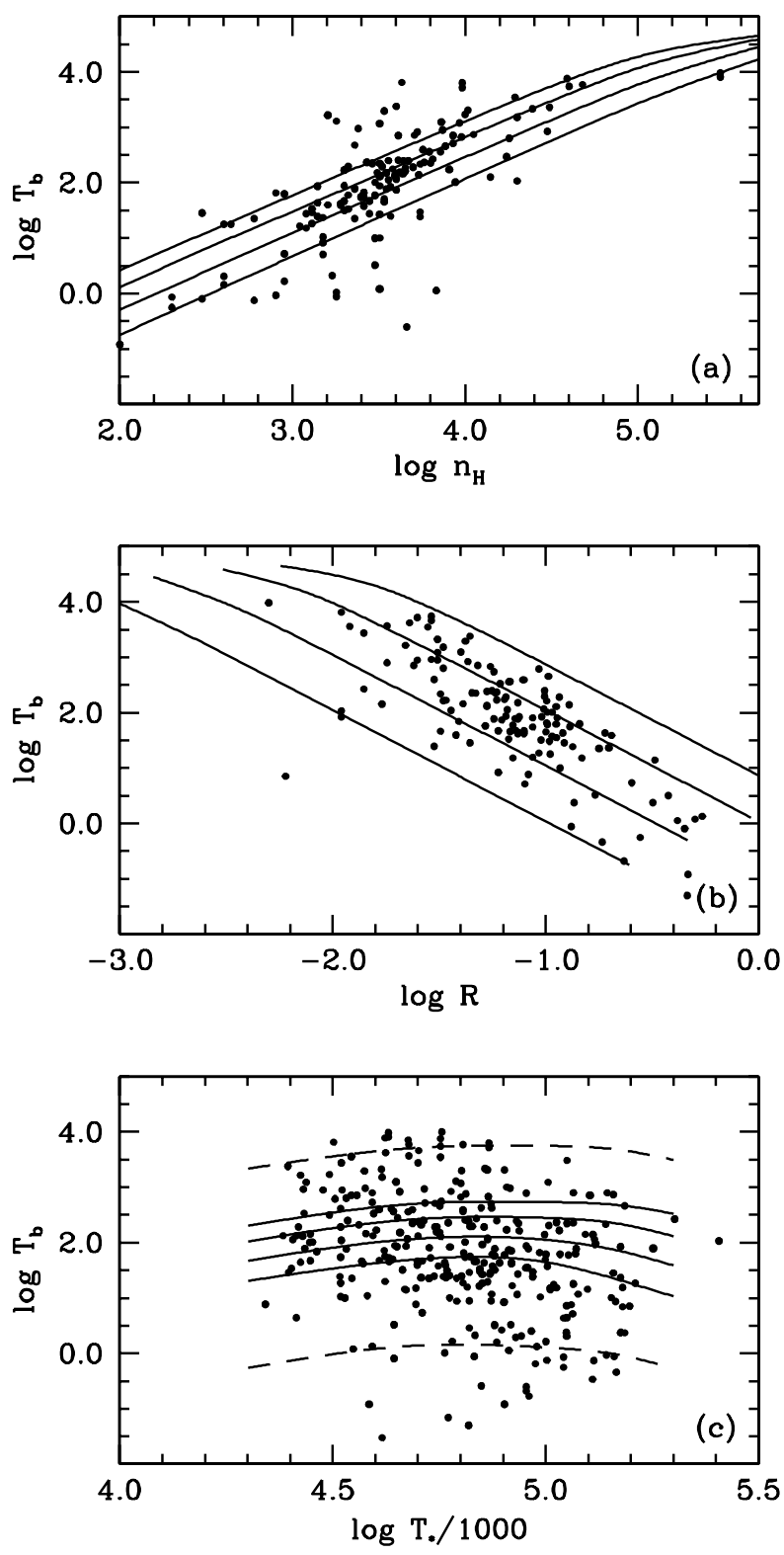

Fig. 4. Radio-continuum brightness temperature in $5 \mathrm{GHz}$, as a function of a) the gas density and $\mathbf{b}$ ) the nebula radius $R$, where higher curves correspond to higher $L_{*}\left(30,300,3000\right.$, and $\left.20000 L_{\odot}\right)$ and $T_{*}=10^{5} \mathrm{~K}$; c) $T_{\mathrm{b}}$ versus $T_{*}$, where solid lines correspond to photoionization models with $n_{\mathrm{H}}=5000 \mathrm{~cm}^{-3}$, for the same values of $L_{*}$ while the dashed lines show the results for $L_{*} / L_{\odot}=3000$ and two values for $n_{\mathrm{H}}(100$ and $50000 \mathrm{~cm}^{-3}$ ), with the higher curve corresponding to a higher $n_{\mathrm{H}}$. Data points are obtained from the literature (see text).

practically negligible (Fig. 4c). As discussed above, this should be expected since for the adopted range of stellar temperatures the number of ionizing photons does not vary much for a given stellar luminosity.

Regarding the effect of the optical thickness, for a model with $T_{*}=10^{5} \mathrm{~K}, L_{*} / L_{\odot}=3000$, and $n_{\mathrm{H}}=5000 \mathrm{~cm}^{-3}$, the brightness temperatures corresponding to a completely optically thick nebula at the Lyman limit, to $\tau_{13.6}=1$, and to $\tau_{13.6}=0.1$ are, respectively, 263, 182, and $94 \mathrm{~K}$. The corresponding fractions of $R_{\max }$ are $1,0.55$, and 0.26 . This shows that the brightness temperature differs significantly from the completely optically thick case only for very small optical depths. 

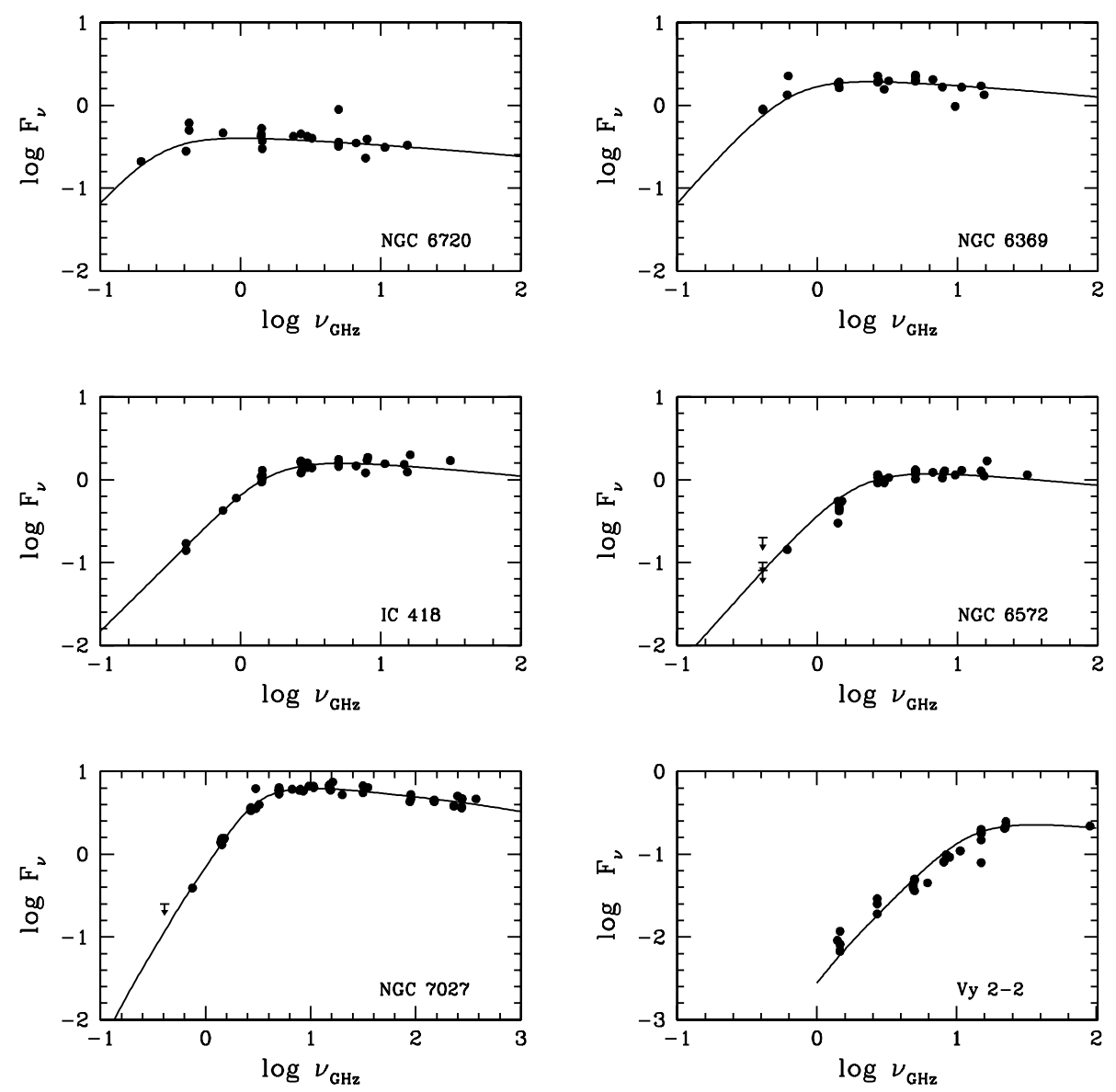

Fig. 5. Radio spectra of some PNe with observed radio spectrum. Data points are from the literature and the curves correspond to photoionization models for uniform density distribution. Flux units are in Jy.

As seen from Figs. 4, the results obtained from models with input parameters for $T_{*}, L_{*}$, and $n_{\mathrm{H}}$ in the typical range known for PNe cover the region defined by the observed points. Figures $4 \mathrm{a}$ and $\mathrm{b}$ present the correlations between $T_{\mathrm{b}}$ and other nebular parameters. In the literature, the accuracy of evolutionary models has been established when the observed data lie within the area covered by the evolutionary model tracks (for example, Zhang \& Kwok 1993; Van de Steene \& Zijlstra 1995). For this, the brightness temperature corresponding to the each nebula is obtained from simplified assumptions for the gas properties. The plots in Figs. 4 show self-consistent results for the brightness temperature, given the nebular gas density, as well as the stellar temperature and luminosity.

\section{Comparing calculated and observed radio spectra}

Using models with a constant density distribution, we compare the calculated and observed radio spectra for some planetary nebulae for which the observed data cover a large range of frequencies. The chosen objects have observed fluxes in the optically thick region of the spectrum as well as in the region around the turn-over frequency, and present spectral indexes over a large range. Points in Fig. 5 represent observed data with flux densities taken from the literature (Menon \& Terzian 1965; Slee \& Orchiston 1965; Terzian 1966; Hughes 1967; Mezger et al. 1967; Thompson et al. 1967; Terzian 1968; Berge \& Seielstad 1969; Kaftan-Kassim 1969; Le Marne 1969; Ribes 1969; Terzian 1969; Thomasson \& Davies 1970;
Table 1. Adopted input parameters for the fitting of the radio spectra.

\begin{tabular}{lrrcc}
\hline \hline Object & $T_{*} /(\mathrm{K})$ & $L_{*} / L_{\odot}$ & $n_{\mathrm{H}}\left(\mathrm{cm}^{-3}\right)$ & $D(\mathrm{kpc})$ \\
\hline IC 418 & 37000 & 9000 & $2.2 \times 10^{4}$ & 1.6 \\
NGC 6369 & 80000 & 6000 & $6.5 \times 10^{3}$ & 1.7 \\
NGC 6572 & 70000 & 8300 & $3.2 \times 10^{4}$ & 2.4 \\
NGC 6720 & 120000 & 200 & $4.0 \times 10^{3}$ & 0.7 \\
NGC 7027 & 300000 & 20000 & $8.0 \times 10^{4}$ & 1.5 \\
Vy 2-2 & 60000 & 10000 & $6.0 \times 10^{5}$ & 5.4 \\
\hline
\end{tabular}

Higgs 1971a,b; Aller \& Milne 1972; Terzian \& Dickey 1973; Cahn \& Rubin 1974; Sistla et al. 1974; Terzian et al. 1974; Milne \& Aller 1975; Harris \& Scott 1976; Marsh et al. 1976; Milne 1979; Milne \& Webster 1979; Cohen \& Barlow 1980; Ulich 1981; Calabretta 1982; Milne \& Aller 1982; Purton et al. 1982; Seaquist \& Davis 1983; Gee et al. 1984; Pottasch 1984; Turner \& Terzian 1984; Gathier et al. 1986; Basart \& Daub 1987; Steppe et al. 1988; Sahai et al. 1989; Hoare et al. 1992; Knapp et al. 1993; Altenhoff et al. 1994; Sandell 1994; Christianto \& Seaquist 1998; Condon \& Kaplan 1998; Bains et al. 2003).

The theoretical spectra shown in Fig. 5 were obtained from photoionization models for uniform spherically symmetric nebulae. The adopted abundances were the same as those of the preceding models, while the adopted stellar temperature and luminosity, as well the gas density for each planetary nebula, are given in Table 1. These values are in the range given by the literature for each object. The presented spectra correspond to optically thick nebulae at the Lyman limit. As seen in Sect. 3.1, 
a moderately matter-bounded nebula would show a spectrum similar to those presented in Fig. 5.

It can be seen that the observed spectra can be reproduced by those from models with a uniform density distribution, when the appropriate variation of the temperature and ionic densities along the nebula are taken into account. No detailed model accounting for the observed emission-line spectra or assuming a particular geometry was searched for each nebula. The purpose of this paper is not to provide detailed models for each nebula. On the contrary, it is our goal to show that a simple model that includes a detailed calculation of the ionizing radiation transfer can explain the radio-continuum observations, including the radio spectral indexes. It is then possible that other sets of input parameters could also fit the spectra. Reasonable fits, similar to those presented in Fig. 5, can also be achieved with other density distributions as, for example, constant pressure, $n_{\mathrm{H}} \propto r^{-1}$, or $\propto r^{-2}$. The gas density is an important parameter defining the spectral behaviour and the radio spectrum is a result of the ionic as well as of the temperature distribution inside the nebula.

Thus, since the density distribution in PNe is not necessarily uniform and more than one density distribution can reproduce the observed data, the radio spectral index does not provide information about the density distribution.

\section{Conclusions}

Radio continuum spectra are obtained for physical conditions typical of PNe, using a photoionization code. The spectra are calculated taking into account self-consistent temperature and ionic distributions, as well as the absorption of the radiation as it crosses the nebula.

The turn-over frequency, as well as the change from the optically thin regime $\left(F_{v} \propto v^{-0.1}\right)$ to the completely optically thick $\left(F_{v} \propto v^{+2.0}\right)$ part of the spectrum, depends on the gas density, with a small effect of the stellar luminosity. Moreover, for a given density, the spectral index of the thick part of the spectrum can span a large range of values depending on the chosen frequency range and on the gas density.

It is shown that self-consistent temperature and ionization distributions throughout the nebula, which result from appropriate radiation transfer calculations, explain the observed range of spectral indexes in the optically thick region of the spectrum, even for a uniform density distribution. The results of photoionization models are compared with those obtained for isothermal and constant ionic density nebulae and show that in the optically thick regime the spectral index is steeper for uniform nebulae.

Defining the spectral index in a frequency range where observed planetary nebulae are commonly observed and are usually thick, we obtained a correlation between the index and the gas density or the nebula radius. Observed correlations between the brightness temperature and some other distance-independent parameters are frequently used to check evolutionary models of the central star and of the nebula. From self-consistent photoionization models we reproduce these correlations in terms of the nebular gas density and the stellar properties.

We present the fitting of radio spectra for some planetary nebulae in a large range of spectral indexes, assuming a uniform density distribution. The facts that the density distribution in $\mathrm{PNe}$ is clearly non-uniform and that other density distributions can also reproduce the observed spectra support the conclusion that the density distribution cannot be inferred only from the radio spectra. Although the obtained results correspond to physical conditions of PNe, the conclusions above are expected also to be valid for ionized gaseous regions in general.
Acknowledgements. We are grateful to S. M. Viegas for interesting discussions and suggestions.

\section{References}

Aaquist, O. B., \& Kwok, S. 1991, ApJ, 378, 599

Aller, L. H., \& Milne, D. K. 1972, Australian J. Phys., 25, 91

Altenhoff, W. J., Thum, C., \& Wendker, H. J. 1994, A\&A, 281, 161

Bains, I., Bryce, M., Mellema, G., Redman, M. P., \& Thomasson, P. 2003, MNRAS, 340, 381

Basart, J. P., \& Daub, C. T. 1987, ApJ, 317, 412

Berge, G. L., \& Seielstad, G. A. 1969, ApJ, 157, 35

Cahn, J. H., \& Rubin, R. H. 1974, AJ, 79, 128

Calabretta, M. R. 1982, MNRAS, 199, 141

Christianto, H., \& Seaquist, E. R. 1998, AJ, 115, 2466

Cohen, M., \& Barlow, M. J. 1980, ApJ, 238, 585

Condon, J. J., \& Kaplan, D. L. 1998, ApJS, 117, 361

Dudziak, G., Pequignot, D., Zijlstra, A. A., \& Walsh, J. R. 2000, A\&A, 363, 717

Gathier, R., Pottasch, S. R., \& Goss, W. M. 1986, A\&A, 157, 191

Gee, G., Emerson, J. P., Ade, P. A. R., Robson, E. I., \& Nolt, I. G. 1984, MNRAS, 208,517

Gruenwald, R., \& Viegas, S. M. 1992, ApJS, 78, 153

Harris, S., \& Scott, P. F. 1976, MNRAS, 175, 371

Higgs, L. A. 1971a, MNRAS, 153, 315

Higgs, L. A. 1971b, Publ. Astrophys. Branch NRC, Canada, 1, 1

Hoare, M. G., Roche, P. F., \& Clegg, R. E. S. 1992, MNRAS, 258, 257

Hughes, M. P. 1967, ApJ, 149, 377

Kaftan-Kassim, M. A. 1969, ApJ, 155, 469

Kingsbourgh, R. L., \& Barlow, R. L. 1994, MNRAS, 271, 257

Knapp, G. R., Sandell, G., \& Robson, E. I. 1993, ApJS, 88, 173

Kwok, S. 1985, AJ, 90, 49

Le Marne, A. E. 1969, Aust. J. Phys., 22, 545

Lynds, C. R. 1961, Pub. Nat. Rad. Astr. Obs., 1, 85

Marsh, K. A. 1975, ApJ, 201, 190

Marsh, K. A., Purton, C. R., \& Felman, P. A. 1976, A\&A, 49, 211

Menon, T. K., \& Terzian, Y. 1965, ApJ, 141, 745

Mezger, P. G., Altenhoff, W., Schraml, J., et al. 1967, ApJ, 150, L157

Middlemass, D. 1990, MNRAS, 244, 294

Milne, D. K. 1979, A\&AS, 36, 227

Milne, D. K., \& Aller, L. H. 1975, A\&A, 38, 183

Milne, D. K., \& Aller, L. H. 1982, A\&AS, 50, 209

Milne, D. K., \& Webster, B. L. 1979, A\&AS, 36, 169

Olnon, F. M. 1975, A\&A, 39, 217

Osterbrock, D. E. 1989, Astrophysics of Gaseous Nebulae and Active Galactic Nuclei (Mill Valley: University Science)

Panagia, N., \& Felli, M. 1975, A\&A, 39, 1

Pottasch, S. R. 1984, Planetary Nebulae: A Study of the Late Stages of Stellar Evolution (Astrophysics and Space Science Library, Dordrecht: Reidel)

Purton, C. R., Feldman, P. A., Marsh, K. A., Allen, D. A., \& Wright, A. E. 1982, MNRAS, 198, 321

Ribes, J. C. 1969, A\&A, 3, 156

Rybicki, G. B., \& Lightman, A. L. 1979, Radiative Processes in Astrophysics (New York: John Wiley \& Sons)

Sahai, R., Claussen, M. J., \& Masson, C. R. 1989, A\&A, 220, 92

Sandell, G. 1994, MNRAS, 271, 75

Seaquist, E. R., \& Davis, L. E. 1983, ApJ, 274, 659

Seaquist, E. R., \& Gregory, P. C. 1973, Nature, 245, 85

Seaton, M. J. 1960, Reports on Progress in Physics, 23, 313

Siodmiak, N., \& Tylenda, R. 2001, A\&A, 373, 1032

Sistla, G., Kojoian, G., \& Chaisson, E. J. 1974, ApJ, 192, 165

Slee, O. B., \& Orchiston, D. W. 1965, Australian J. Phys., 18, 187

Stasinska, G., \& Tylenda, R. 1986, A\&A, 155, 137

Steppe, H., Salter, C. J., Chini, R., et al. 1988, A\&AS, 75, 317

Taylor, A. R., Pottasch, S. R., \& Zhang, C. Y. 1987, A\&A, 171, 178

Terzian, Y. 1966, ApJ, 144, 657

Terzian, Y. 1968, Planetary Nebulae, ed. Osterbrock, \& O’Dell, IAU, 34, 87

Terzian, Y. 1969, Astrophys. Lett., 3, 87

Terzian, Y., \& Dickey, J. 1973, AJ, 78, 875

Terzian, Y., Balick, B., \& Bignell, C. 1974, ApJ, 188, 257

Thomasson, P., \& Davies, J. G. 1970, MNRAS, 150, 359

Thompson, A. R., Colvin, R. S., \& Stanley, G. J. 1967, ApJ, 148, 429

Turner, K. C., \& Terzian, Y. 1984, AJ, 89, 501

Ulich, B. L. 1981, AJ, 86, 1619

Van de Steene, G. C., \& Zijlstra, A. A. 1995, A\&A, 293, 541

Viner, M. R., Vallee, J. P., \& Hughes, V. A. 1979, ApJS, 39, 405

Wright, A. E., \& Barlow, M. J. 1975, MNRAS, 170, 41

Zhang, C. Y. 1995, ApJS, 98, 659

Zhang, C. Y., \& Kwok, S. 1993, ApJS, 88, 137 